International Journal of Pure and Applied Mathematics

Volume 109 No. 2 2016, 193-205

ISSN: 1311-8080 (printed version); ISSN: 1314-3395 (on-line version)

url: http://www.ijpam.eu

doi: 10.12732/ijpam.v109i2.3

\title{
ON NEW ITERATION FOR SET CONTRACTION MAPPINGS AND EXISTENCE RESULTS FOR POWER SET CONTRACTIONS MAPPINGS
}

\author{
Nour El Houda Bouzara ${ }^{1 \S}$, Vatan Karakaya ${ }^{2}$ \\ ${ }^{1,2}$ Department of Mathematics \\ Yildiz Technical University \\ Davutpasa Campus \\ Esenler, Istanbul, 34220, TURKEY
}

\begin{abstract}
In this paper, we present a new iteration for set contraction mappings in a Banach space. In further, we introduce the concept of set stability for these class of mappings. Finally, we present some results on the existence of fixed points for power set contraction mappings, these results include in special case many results existing in literature like Darbo and generalized Darbo fixed point theorems.
\end{abstract}

AMS Subject Classification: 47H10, 47H08

Key Words: set contraction, measure of noncompactness, fixed point, iteration, stability

\section{Introduction}

In the last years, measure of noncompactness revealed to be very useful in resolving different differential and integral equations (see $[2-4,6,13,22]$ and many others). It is considered as a tool to get over the problem of lack of compactness in fixed point theorems. This appears clearly in the Darbo fixed point theorem [8] where we use condensing instead of compact mappings. That is remain to say that the Darbo fixed point theorem makes a combination of two classical theorems in the fixed point theory : Schauder fixed point theorem [21] and Banach Contraction Principal [1]. However, it does guarantee only the

Received: March 8, 2016

Published: August 31, 2016

${ }^{\S}$ Correspondence author (c) 2016 Academic Publications, Ltd.

url: www.acadpubl.eu 
existence and not the unicity of fixed points or a way to reach them.

Our aim in this paper is to introduce an iteration that converges to the set of fixed points for set contraction mappings. Also, establish results of stability for these type of mappings. Finally, present some results on the existence of fixed points for power set contraction mappings, these results include in special case many results existing in literature.

\section{Preliminaries}

Throughout this paper, we suppose that $(X,\|\|$.$) is a real Banach space and$ $\mathcal{M}_{X}$ the collection of all nonempty bounded subsets of $X$. co $(A)$ denotes the convex hull of a set $A$.

Let $A$ be a bounded subset of a Banach space $X$. The Kuratowski measure of noncompactness [15] is defined by

$$
\alpha(A)=\inf \{d>0:
$$

$A$ is covered by a finite number of sets with diameter $\leqslant d\}$.

It is clear that $0 \leqslant \alpha(A)<\infty$.

If instead of sets we use balls we get the Hausdorff measure of noncompactness [2]

$$
\beta(A)=\inf \{d>0:
$$

$A$ is covered by a finite number of balls with diameter $\leqslant d\}$.

The relation between Kuratowski and Hausdorff measure of noncompactness is given by the following inequalities

$$
\beta(A) \leqslant \alpha(A) \leqslant 2 \beta(A) .
$$

Remark $1([4])$.

1. The diameter of a set $A$ is the number $\sup \{d(x, y): x \in A, y \in A\}$ denoted by $\operatorname{diam}(A)$. It is clear that

$$
0 \leqslant \alpha(A) \leqslant \operatorname{diam}(A)<+\infty .
$$

If $\operatorname{diam}(A)=0$, then $\alpha(A)=0$. But we know that $\operatorname{diam}(A)=0$ if and only if $A$ is an empty set or consists of exactly one point and these both cases correspond to $\alpha(A)=0$. 
2. Let $\mu$ any measure of noncompactness, the following properties are satisfied in any complete metric space $X$ and are a direct consequence of the definition:

(a) $\mu(A)=0 \Leftrightarrow A$ is a precompact set.

(b) $\mu(A)=\mu(\bar{A}), \forall A \in \mathcal{B}_{X}$.

(c) $\mu(A \cup B)=\max (\mu(A), \mu(B)), \forall A, B \in \mathcal{B}_{X}$.

(d) $\mu(A \cap B)=\inf (\mu(A), \mu(B)), \forall A, B \in \mathcal{B}_{X}$.

(e) $A \subset B \Rightarrow \mu(A) \leqslant \mu(B), \forall A, B \in \mathcal{B}_{X}$.

(f) If $A$ is a finite set, then $\mu(A)=0$.

(g) $\mu$ is continuous with respect to Hausdorff metric.

3. The following properties are satisfied in any complete normed space:

(a) $\mu(\lambda A)=|\lambda| \mu(A)$, where $\lambda \in \mathbb{R}$ and $\lambda A=\{\lambda x: x \in A\}$.

(b) $\mu(A+B) \leqslant \mu(A)+\mu(B)$, where $A+B=\{x+y: x \in A$ and $y \in B\}$. In particular, if $A=\left\{x_{n}\right\}$ and $B=\left\{y_{n}\right\}$ are two countable of sets of points in $X$, then

$$
\mu\left(\left\{x_{n}\right\}\right)-\mu\left(\left\{y_{n}\right\}\right) \leqslant \mu\left(\left\{x_{n}-y_{n}\right\}\right) .
$$

(c) For any $\lambda \in(0,1)$, we have

$$
\mu(\lambda A+(1-\lambda) B) \leqslant \lambda \mu(A)+(1-\lambda) \mu(B) .
$$

(d) $\mu(\operatorname{Conv} A)=\mu(A), \forall A, B \in \mathcal{B}_{X}$.

Lemma 1 ([16]). Let $A_{n}$ be a sequence of subsets of $X$ such that $A_{n}$ approaches a subset $A_{\infty}$ in the Hausdorff metric. Then, if $A_{n}$ are bounded, we have $\lim _{n \rightarrow \infty} \mu\left(A_{n}\right)=\mu\left(A_{\infty}\right)$

The concept of measure of noncompactness was the first time was introduced by Kuratowski in order to generalize the Cantor intersection theorem.

Theorem 1 (Kuratowski [14]). Let $(X, d)$ be a complete metric space and $\left(A_{n}\right)_{n}$ be a decreasing sequence of nonempty, closed subsets of $\mathcal{B}_{X}$ such that $\lim _{n \rightarrow \infty} \mu\left(A_{n}\right)=0$. Then, the intersection set $A_{\infty}=\bigcap_{n=1}^{\infty} A_{n}$ is nonempty and compact. 
In the following a new more simple proof of the previous theorem by using the continuity of measure of noncompactness.

Proof. To prove this, we use the property $(d)$,

$$
\mu\left(\bigcap_{i=1}^{n} A_{i}\right)=\inf \left(\mu\left(A_{i}\right), i=1, \ldots, n\right) \leqslant \mu\left(A_{n}\right)
$$

By taking the limits we get,

$$
\lim _{n \rightarrow \infty} \mu\left(\bigcap_{i=1}^{n} A_{i}\right) \leqslant \lim _{n \rightarrow \infty} \mu\left(A_{n}\right)=0 .
$$

Then,

$$
\lim _{n \rightarrow \infty} \mu\left(\bigcap_{i=1}^{n} A_{i}\right)=0
$$

Since the measure of noncompactness is continuous, then

$$
\begin{aligned}
\lim _{n \rightarrow \infty} \mu\left(\bigcap_{i=1}^{n} A_{i}\right) & =\mu\left(\lim _{n \rightarrow \infty} \bigcap_{i=1}^{n} A_{i}\right) \\
& =\mu\left(\bigcap_{i=1}^{\infty} A_{i}\right) \\
& =0 .
\end{aligned}
$$

Theorem 2 (Banach Contraction Theorem [1]). Let $(Y, d)$ be a complete metric space and $f: Y \rightarrow Y$ be a map such that

$$
d(f x, f y) \leqslant k d(x, y)
$$

for some $0 \leqslant k<1$ and all $x, y$ in $Y$. Then, $f$ has a unique fixed point in $Y$.

Moreover, for any $x_{0} \in Y$ the sequence of iterates $x_{n+1}=f\left(x_{n}\right)$ converges to the fixed point of $f$.

Theorem 3 (Schauder [21]). Every continuous self-mapping on a compact subset of a Banach space has a fixed point. 


\section{Main Results}

\subsection{Convergence Results}

Theorem 4. Let $A$ be a nonempty closed, bounded and convex subset of $X$. If $N: A \rightarrow A$ is a continuous mapping such that

$$
\mu(N A) \leqslant k \mu(A), \quad k \in[0,1)
$$

then $N$ has a fixed point in $A$.

Moreover, let the closed bounded convex sequence $\left(A_{n}\right)_{n \in N}$, then for any $A_{1}$, the sequence of subsets $A_{n+1}=c o\left(N A_{n}\right)$ converges to the set of fixed points of $N$.

Proof. The existence part is given by Darbo fixed point theorem [2].

In the next we prove the convergence of the iteration $A_{n+1}=\operatorname{co}\left(N A_{n}\right)$,

$$
\begin{aligned}
\left\|\mu\left(A_{n+1}\right)-\mu(\mathcal{F})\right\| & =\left\|\mu\left(N A_{n}\right)-\mu(N \mathcal{F})\right\| \\
& \leqslant\left\|k \mu\left(A_{n}\right)-k \mu(\mathcal{F})\right\| \\
& \leqslant k\left\|\mu\left(A_{n}\right)-\mu(\mathcal{F})\right\|
\end{aligned}
$$

In further,

$$
\begin{aligned}
\left\|\mu\left(A_{n}\right)-\mu(\mathcal{F})\right\| & =\left\|\mu\left(N A_{n-1}\right)-\mu(N \mathcal{F})\right\| \\
& \leqslant k\left\|\mu\left(A_{n-1}\right)-\mu(\mathcal{F})\right\| .
\end{aligned}
$$

Substituting (3.2) in (3.1), we get

$$
\left\|\mu\left(A_{n+1}\right)-\mu(\mathcal{F})\right\| \leqslant k^{2}\left\|\mu\left(A_{n-1}\right)-\mu(\mathcal{F})\right\| .
$$

Repeating this process $n-1$ time, we get

$$
\left\|\mu\left(A_{n+1}\right)-\mu(\mathcal{F})\right\| \leqslant k^{n}\left\|\mu\left(A_{1}\right)-\mu(\mathcal{F})\right\| .
$$

Since $k \in\left[0,1\left[, \lim _{n \rightarrow \infty} k^{n}=0\right.\right.$, then

$$
\lim _{n \rightarrow \infty}\left\|\mu\left(A_{n+1}\right)-\mu(\mathcal{F})\right\|=0
$$

Thus, $\lim _{n \rightarrow \infty} \mu\left(A_{n+1}\right)=\mu(\mathcal{F})$ and using the fact that $\mu$ is continuous with respect to Hausdorff metric, then $\lim _{n \rightarrow \infty} A_{n+1}=\mathcal{F}$. 
Remark 2. These results doesn't remind true for metric spaces unless if the metric verify the following inequality,

$$
d(k x, k y) \leqslant k d(x, y) .
$$

Corollary 1. Let $X$ be a Banach space and a mapping $T: X \rightarrow X$ such that

$$
\operatorname{diam}(T A) \leqslant k \cdot \operatorname{diam}(A) .
$$

Then, $T$ has a unique fixed point in $X$.

Proof. Let the iteration $A_{n+1}=\operatorname{conv}\left(T A_{n}\right)$ and by using the properties of diameter we get

$$
\begin{aligned}
\operatorname{diam}\left(A_{n+1}\right) & =\operatorname{diam}\left(\operatorname{conv}\left(T A_{n}\right)\right) \\
& =\operatorname{diam}\left(T A_{n}\right) \\
& \leqslant \operatorname{diam}\left(A_{n}\right)
\end{aligned}
$$

Repeating this process $n$-times we get

$$
\operatorname{diam}\left(A_{n+1}\right) \leqslant k^{n} \operatorname{diam}\left(A_{1}\right) .
$$

Since $k \in[0,1)$, then $\lim _{n \rightarrow \infty} k^{n}=0$. Thus, $\lim _{n \rightarrow \infty} \operatorname{diam}\left(A_{n+1}\right)=0$ or using the fact that $\lim _{n \rightarrow \infty} A_{n+1}=\mathcal{F}, \operatorname{diam}(\mathcal{F})=0$.

We conclude that either the set of fixed points of $\mathcal{F}$ is empty or contains only one point. However, it couldn't be empty since $T$ is k-set contraction, hence $\mathcal{F}$ contains only one point.

Remark 3. The above corollary includes the Banach contraction principle as a special case. Indeed, suppose that $T$ is a contraction mapping, then

$$
\|T x-T y\| \leqslant k\|x-y\|, \text { for any } x, y \in X .
$$

In further, we know that the diameter is the simplest measure of noncompactness and by definition for any bounded set $A, \operatorname{diam}(A)=\sup _{x, y \in A}\|x-y\|$.

By taking supremum in Inequality 3.3. we get

$$
\sup _{T x, T y \in T A}\|T x-T y\| \leqslant k \sup _{x, y \in A}\|x-y\| .
$$

Then,

$$
\operatorname{diam}(T A) \leqslant k \operatorname{diam}(A) .
$$




\subsection{Stability Results}

The concept of stability was introduced by Harder [9], Harder and Hicks [10, 11]. Roughly speaking a fixed point iteration procedure is numerically stable if by effecting small modifications in initial data involved in the computation process we get a small influence on the computed value of the fixed point. There are also other definitions of stability considered by several authors, for example : Berinde [5], Imoru and Olatinwo [12], Osilike [17], Osilike and Udomene [18], Rhoades $[19,20]$ and many others.

Inspired by the definition given by Harder and Hicks, we introduce the following definition:

Definition 1. Let $N$ be a $k$-set contraction mapping and $\left(A_{n}\right)$ is a sequence of nonempty, closed, bounded and convex subset of $X$ such that $A_{n+1}=c o\left(N A_{n}\right)$ and converges to $\mathcal{F}$ (the set of fixed points of $\left.N\right)$. Let $\epsilon_{n}=\left\|\mu\left(B_{n+1}\right)-\mu\left(f\left(N, A_{n}\right)\right)\right\|$, where $\left(B_{n}\right)$ is a sequence of nonempty, closed, bounded and convex subsets of $X$ such that $B_{n+1}=c o\left(N B_{n}\right)$. The mapping $N$ is said to be $N$-set stable if the following assumption holds,

$$
\lim _{n \rightarrow \infty} \epsilon_{n}=0 \Longleftrightarrow \lim _{n \rightarrow \infty} A_{n+1}=\lim _{n \rightarrow \infty} B_{n+1}=\mathcal{F} \text {. }
$$

Theorem 5. Let $N$ be a $k$-set contraction mapping and let the iteration $A_{n+1}=c o\left(N A_{n}\right)$ where $\left(A_{n}\right)$ is a sequence of nonempty, closed, bounded and convex subsets of $X$. Then, the mapping $N$ is set stable.

Proof. Suppose that $\lim _{n \rightarrow \infty} \epsilon_{n}=0$, and let show that

$$
\lim _{n \rightarrow \infty} A_{n+1}=\lim _{n \rightarrow \infty} B_{n+1}=\mathcal{F} \text {. }
$$

We have

$$
\begin{aligned}
\left\|\mu\left(B_{n+1}\right)-\mu(\mathcal{F})\right\| & =\left\|\mu\left(B_{n+1}\right)-\mu\left(A_{n+1}\right)+\mu\left(A_{n+1}\right)-\mu(\mathcal{F})\right\| \\
& \leqslant\left\|\mu\left(B_{n+1}\right)-\mu\left(A_{n+1}\right)\right\|+\left\|\mu\left(A_{n+1}\right)-\mu(\mathcal{F})\right\|,
\end{aligned}
$$

since,

$$
\lim _{n \rightarrow \infty} \epsilon_{n}=\lim _{n \rightarrow \infty}\left\|\mu\left(B_{n+1}\right)-\mu\left(A_{n+1}\right)\right\|=0
$$

and

$$
\lim _{n \rightarrow \infty}\left\|\mu\left(A_{n+1}\right)-\mu(\mathcal{F})\right\|=0 \text {, then } \lim _{n \rightarrow \infty}\left\|\mu\left(B_{n+1}\right)-\mu(\mathcal{F})\right\|=0 .
$$

On the other hand, suppose that $\lim _{n \rightarrow \infty} B_{n+1}=\mathcal{F}$ and let show that $\lim _{n \rightarrow \infty} \epsilon_{n}=0$. 
Let,

$$
\begin{aligned}
\left\|\mu\left(B_{n+1}\right)-\mu\left(A_{n+1}\right)\right\| & =\left\|\mu\left(B_{n+1}\right)-\mu(\mathcal{F})+\mu(\mathcal{F})-\mu\left(A_{n+1}\right)\right\| \\
& \leqslant\left\|\mu\left(B_{n+1}\right)-\mu(\mathcal{F})\right\|+\left\|\mu\left(A_{n+1}\right)-\mu(\mathcal{F})\right\| .
\end{aligned}
$$

By taking the limit, we get

$$
\lim _{n \rightarrow \infty} \epsilon_{n}=\lim _{n \rightarrow \infty}\left\|\mu\left(B_{n+1}\right)-\mu\left(A_{n+1}\right)\right\|=0 .
$$

This ends the proof.

\subsection{Existence of Fixed Points for Power Set Contraction Mappings}

We know that a contraction mapping $N$ on a Banach space $(X,\|\cdot\|)$ with contraction constant $k$, is also a contraction on $X$ with a contraction constant $k^{n}$. Then, what we can say about a set contraction mappings?!!

Theorem 6. Let $A$ be a nonempty closed, bounded and convex subspace of a Banach space $(X,\|\|$.$) and N: A \rightarrow A$ be a $k$-set contraction mapping on $A$. Then, $N^{n}$ (for an integer $n>0$ ) is a $k^{n}$-set contraction on $A$.

Proof. Let $A$ be a nonempty closed, bounded and convex subset of $X$, then

$$
\begin{aligned}
\mu\left(N^{n} A\right) & =\mu\left(N\left(N^{n-1} A\right)\right) \\
\leqslant & k \mu\left(N^{n-1} A\right) \\
\leqslant & k^{2} \mu\left(N^{n-2} A\right) \\
& \vdots \\
\leqslant & k^{n} \mu(A)
\end{aligned}
$$

Since $0 \leqslant k<1$, hence $0 \leqslant k^{n}<1$ and so $N^{n}$ is a $k$-set contraction mapping.

Remark 4. The inverse is not true that is if $N^{n}$ is a $k$-set contraction mapping then $N$ could be not a $k$-set contraction mapping.

Example 1. Let $N: X \rightarrow X$, where $X$ is a Banach space, be a mapping defined by $N x=1-\frac{x}{2}$ for any $x \in X$.

Let $B(0, r)$ be an open ball with center 0 and radius $r \leqslant 1$. Then,

$$
N B(0, r)=B\left(0,1-\frac{r}{2}\right)
$$


It is easy to see that $B\left(0,1-\frac{r}{2}\right) \nsubseteq B(0, r)$, hence $N$ isn't a $k$-set contraction mapping.

However, $N^{2} x=N\left(1-\frac{x}{2}\right)=\frac{x}{2}$, then

$$
N^{2} B(0, r)=B\left(0, \frac{r}{2}\right) \subseteq B(0, r)
$$

Thus for any measure of noncompactness $\mu$ we have

$$
\mu\left(N^{2} B(0, r)\right) \leqslant \frac{1}{2} \mu(B(0, r))
$$

hence $N^{2}$ is a $k$-set contraction mapping with $k=\frac{1}{2}$.

Theorem 7. Let $A$ be a nonempty closed, bounded and convex subspace of a Banach space $(X,\|\|$.$) and N: A \rightarrow A$ be a mapping such that for any $n \geqslant 1$ we have $N^{n}(\operatorname{conv}(A)) \subseteq \operatorname{conv}\left(N^{n} A\right)$ and

$$
\mu\left(N^{n} A\right) \leqslant k_{n} \mu(A)
$$

where $k_{n} \rightarrow 0, n \rightarrow+\infty$. Then, $N$ has at least one fixed point.

Proof. Let the iteration $A_{n}=\operatorname{conv}\left(N A_{n-1}\right)$, where $\left(A_{n}\right)$ is a sequence of nonempty closed, bounded nd convex subsets of $X$.

It is clear that $\left(A_{n}\right)_{n}$ is decreasing and by using the properties of the measure of noncompactness, we get

$$
\begin{aligned}
\mu\left(A_{n}\right) & =\mu\left(\operatorname{conv}\left(N A_{n-1}\right)\right) \\
& \leqslant \mu\left(\operatorname{conv}\left(N A_{n-1}\right)\right)=\mu\left(N A_{n-1}\right) \\
& \leqslant \mu\left(N\left(\operatorname{conv}\left(N A_{n-2}\right)\right)\right) \\
& \leqslant \mu\left(N\left(\operatorname{conv}\left(N A_{n-2}\right)\right)\right) \\
& \leqslant \mu\left(N^{2}\left(A_{n-2}\right)\right)
\end{aligned}
$$

Repeating this process many times we get

$$
\mu\left(A_{n}\right) \leqslant \mu\left(N^{n}\left(A_{0}\right)\right)
$$

Using Inequality 3.4. we get $\mu\left(A_{n}\right) \leqslant \mu\left(N^{n}\left(A_{0}\right)\right) \leqslant k_{n} \mu\left(A_{0}\right)$.

By taking the limit, we get $\lim _{n \rightarrow \infty} \mu\left(A_{n}\right)=0$, which implies that $A_{\infty}$ is compact and hence $N$ has at least one fixed point in $A_{\infty}$. 
Corollary 2. Let $X$ be a Banach space and $N$ be a mapping such that for each $n \geqslant 1$, there exists a constant $k_{n}$ such that

$$
\sup _{N^{\mathrm{n}} x, N^{\mathrm{n}} y \in N^{\mathrm{n}} A}\left\|N^{n} x-N^{n} y\right\| \leqslant k_{n} \sup _{x, y \in A}\|x-y\| \text { for all } A \in \mathcal{M}_{X},
$$

where, $k_{n} \rightarrow 0, n \rightarrow+\infty$. Then, $N$ has a unique fixed point.

Proof. Easy to see, since by definition $\operatorname{diam}(A)=\sup _{x, y \in A}\|x-y\|$.

The above corollary includes the theorem given by Caccioppoli in [7] as a special case.

Theorem 8 (Caccioppoli). Let $N$ be a mapping such that for each $n \geqslant 1$, there exists a constant $k_{n}$ such that

$$
\left\|N^{n}(x)-N^{n}(y)\right\| \leqslant k_{n}\|x-y\| \text { for all } u, v \in X,
$$

where, $\sum_{n=1}^{\infty} k_{n}<\infty$. Then, $N$ has a unique fixed point.

Proof. As we know the simplest measure of noncompactness is the diameter

$$
\operatorname{diam}=\sup _{u, v \in A} d(u, v) .
$$

Let,

$$
d\left(N^{n}(u), N^{n}(v)\right) \leqslant k_{n} d(u, v) .
$$

Then,

$$
\sup d\left(N^{n}(u), N^{n}(v)\right) \leqslant k_{n} \sup d(u, v) .
$$

This is equivalent to say that

$$
\mu\left(N^{n} A\right) \leqslant k_{n} \mu(A) .
$$

However, since $\sum_{n=1}^{\infty} k_{n}<\infty$, then $k_{n} \rightarrow 0$ for $n \rightarrow \infty$.

Then from Theorem 7. $N$ has at least one fixed point.

Suppose that $p$ and $q$ are two fixed points for $N$. Then,

$$
d(p, q)=d\left(N^{n}(p), N^{n}(q)\right) \leqslant k_{n} d(p, q)
$$

Using that $k_{n} \rightarrow 0, n \rightarrow \infty$. We obtain

$$
0 \leqslant d(p, q) \leqslant 0
$$

thus $q=p$ and the fixed point is unique. 
Theorem 9. Let $A$ be a bounded subset of $\mathrm{a}(X,\|\cdot\|)$ be a Banach space and $N: A \rightarrow A$ satisfies

$$
\mu\left(N^{n} A\right) \leqslant \eta(\mu(A)) \mu(A),
$$

where, either $\eta: \mathbb{R}_{+} \rightarrow[0,1)$ is a decreasing function or $\eta: \mathbb{R}_{+} \rightarrow[1, \infty)$ is a function such that $\lim _{n \rightarrow \infty} \eta\left(t_{n}\right)=1$ implies $\lim _{n \rightarrow \infty} t_{n}=0$.

Then, $N$ has a fixed point in $A$.

Proof. Let $A_{n+1}=\operatorname{conv}\left(N^{n} A_{n}\right)$ such that $\left(A_{n}\right)$ be a sequence of nonempty closed, bounded and convex subsets of a Banach space $X$. It is easy to see that $\left(A_{n}\right)$ is a decreasing sequence.

In further, by using Condition 3.5. we get

$$
\mu\left(A_{n+1}\right) \leqslant \eta\left(\mu\left(A_{n}\right)\right) \mu\left(A_{n}\right) .
$$

Suppose that $\eta: \mathbb{R}_{+} \rightarrow[1, \infty)$ is a function such that $\lim _{n \rightarrow \infty} \eta\left(t_{n}\right)=1$ implies $\lim _{n \rightarrow \infty} t_{n}=0$.

In further, since $\left(A_{n}\right)$ is a decreasing sequence

$$
1 \leqslant \frac{\mu\left(A_{n}\right)}{\mu\left(A_{n+1}\right)} \leqslant \frac{1}{\eta\left(\mu\left(A_{n}\right)\right)} \leqslant 1 .
$$

By taking limit, we obtain

$$
\lim _{n \rightarrow \infty} \mu\left(A_{n}\right)=\lim _{n \rightarrow \infty} \mu\left(A_{n+1}\right) \text { and } \lim _{n \rightarrow \infty} \frac{1}{\eta\left(\mu\left(A_{n}\right)\right)}=1 .
$$

Hence, $\lim _{n \rightarrow \infty} \eta\left(\mu\left(A_{n}\right)\right)=1$, which implies that $\lim _{n \rightarrow \infty} \mu\left(A_{n}\right)=0$. Thus, $A_{\infty}$ is compact and $N$ has at least one fixed point in $A_{\infty}$.

Now, suppose that $\eta: \mathbb{R}_{+} \rightarrow[0, \infty)$ is a decreasing function.

From Inequality 3.6. we have

$$
0 \leqslant \frac{\mu\left(A_{n+1}\right)}{\mu\left(A_{n}\right)} \leqslant \eta\left(\mu\left(A_{n}\right)\right)
$$

Repeating this process and using the fact that $\eta$ is a decreasing function, we obtain

$$
0 \leqslant \frac{\mu\left(A_{n}\right)}{\mu\left(A_{n-1}\right)} \leqslant \eta\left(\mu\left(A_{n-1}\right)\right) \leqslant \eta\left(\mu\left(A_{n}\right)\right) .
$$




$$
0 \leqslant \frac{\mu\left(A_{1}\right)}{\mu\left(A_{0}\right)} \leqslant \eta\left(\mu\left(A_{0}\right)\right) \leqslant \eta\left(\mu\left(A_{n}\right)\right) .
$$

In further, we have

$$
\begin{aligned}
0 \leqslant \frac{\mu\left(A_{n+1}\right)}{\mu\left(A_{0}\right)} & =\frac{\mu\left(A_{n+1}\right)}{\mu\left(A_{n}\right)} \cdot \frac{\mu\left(A_{n}\right)}{\mu\left(A_{n-1}\right)} \ldots \frac{\mu\left(A_{1}\right)}{\mu\left(A_{0}\right)} \\
& \leqslant \eta\left(\mu\left(A_{n}\right)\right) \cdot \eta\left(\mu\left(A_{n}\right)\right) \ldots \eta\left(\mu\left(A_{n}\right)\right) \\
& =\left(\eta\left(\mu\left(A_{n}\right)\right)\right)^{n} .
\end{aligned}
$$

Since $\eta\left(\mu\left(A_{n}\right)\right) \in[0,1)$, then $\lim _{n \rightarrow \infty}\left(\eta\left(\mu\left(A_{n}\right)\right)\right)^{n}=0$ and so $\lim _{n \rightarrow \infty} \frac{\mu\left(A_{n}+1\right)}{\mu\left(A_{0}\right)}=0$ that is $\lim _{n \rightarrow \infty} \mu\left(A_{n+1}\right)=0$ since $\mu\left(A_{0}\right)$ is a finite constant.

Consequently, $A_{\infty}$ is compact and $N$ has a fixed point in $A_{\infty}$.

Theorem 10. Let $A$ be a nonempty closed, bounded and convex subspace of a Banach space $(X,\|\|$.$) and N: A \rightarrow A$ be a mapping such that for any $n \geqslant 1$ we have $N^{n}(\operatorname{conv}(A)) \subseteq \operatorname{conv}\left(N^{n} A\right)$ and

$$
\mu\left(N^{n} A\right) \leqslant \varphi_{n}(\mu(A))
$$

where $\varphi_{n}:[0, \infty) \rightarrow[0, \infty)$ are continuous and $\varphi_{n} \rightarrow 0, n \rightarrow+\infty$ uniformly. Then, $N$ has at least one fixed point in $A$.

Proof. Let $A_{n+1}=\operatorname{conv}\left(N A_{n}\right)$ such that $\left(A_{n}\right)$ be a sequence of nonempty closed, bounded and convex subsets of a Banach space $X$. then as we did in 3.3 , we get

$$
\mu\left(A_{n}\right) \leqslant \mu\left(N^{n}\left(A_{0}\right)\right) .
$$

By Condition 3.7. we obtain

$$
\mu\left(A_{n}\right) \leqslant \varphi_{n}\left(\mu\left(A_{0}\right)\right)
$$

By taking limits, we get

$$
0 \leqslant \lim _{n \rightarrow \infty} \mu\left(A_{n}\right) \leqslant \lim _{n \rightarrow \infty} \varphi_{n}\left(\mu\left(A_{0}\right)\right)=0
$$

Thus, $\lim _{n \rightarrow \infty} \mu\left(A_{n}\right)=0$. Hence, $A_{\infty}$ is compact and $N$ has at least one fixed point in $A_{\infty}$. 


\section{References}

[1] R. Agarwal, M. Meehan, D. O'Regan, Fixed Point Theory and Applications, Cambridge University Press, Cambridge (2001). doi:10.1017/CBO9780511543005

[2] R. R. Akmerov, M. I. Kamenski, A. S. Potapov, A. E. Rodkina, B. N. Sadovskii, Measures of Noncompactness and Condensing Operators, Birkhauser-Verlag, Basel (1992). doi:10.1007/978-3-0348-5727-7.

[3] J. Apell, Measure of noncompactness, condensing operator and fixed points: An application-oriented survey, Fixed Point Theory, 6 (2005), 157-229.

[4] J. M. Ayerbe Toledano, T. Dominguez Benavides, G. López Acedo, Measures of Noncompactness in Metric Fixed Point Theory, Birkhauser Verlag (1997). doi:10.1007/9783-0348-8920-9.

[5] V. Berinde, On the stability of some fixed point procedures, Bul. Stiint. Univ. Baia Mare, Ser. B, Mat-Inform., 18, No. 1 (2002), 7-14.

[6] J. Banaś, On measures of noncompactness in Banach spaces, Comment. Math. Univ. Carolin. 21 (1980), 131-143.

[7] R. Caccioppoli, Un teorema generale sullésistenza de elemente uniti in una transformazione funzionale, Rend. Acad. Naz. Linzei, 11 (1930), 31-49.

[8] G. Darbo, Punti unitti in transformazioni a condominio non compatto, Rend. Semin. Mat. Univ. Padova, 24 (1955), 84-92.

[9] A. M. Harder, Fixed point theory and stability results for fixed point iteration procedures, Ph.D. Thesis, University of Missouri-Rolla, Missouri (1987).

[10] A. M. Harder, T. L. Hicks, A stable iteration procedure for nonexpansive mappings, Math. Japon, 33 (1988), 687-692.

[11] A. M. Harder, T. L. Hicks, Stability results for fixed point iteration procedures, Math. Japon., 33 (1988), 693-706.

[12] C. O. Imoru, M. O. Olatinwo, On the stability of Picard and Mann iteration processes, Carpathian J. Math., 19 (2003), 155-160.

[13] V. Karakaya, N. H. Bouzara, K. Dogan, Y. Atalan, Existence of tripled fixed points for a class of condensing operators in Banach spaces, TheScientific WorldJournal., 2014 (2014), 1-9. doi:10.1155/2014/541862.

[14] W. A. Kirk, B. Sims, Handbook of metric fixed point theory, Kluwer Academic Publishers (2001). doi:0.1007/978-94-017-1748-9

[15] K. Kuratowski, Sur les espaces complets, Fund. Math. 5 (1930), 301-309.

[16] V. Lakshmikantham, S. Leela, Z. Drici, F. A. McRae, Theory of Causal Differential Equations, Atlantis Press (2009). doi.org/10.2991/978-94-91216-25-1

[17] M. O Osilike, Stability results for the Ishikawa fixed point iteration procedure, Indian J. Pure Appl. Math., 26 (1995), 937-945.

[18] M. O. Osilike, A. Udomene, Short proofs of stability results for fixed point iteration procedures for a class of contractive type mappings, Indian J. Pure Appl. Math, 30, No 12 (1999), 1229-1234.

[19] B. E. Rhoades, Fixed point theorems and stability results for fixed point iteration procedures, Indian J. Pure Appl. Math, 21 (1990), 1-9. 
[20] B. E. Rhoades, Fixed point theorems and stability results for fixed point iteration procedures II, Indian J. Pure Appl. Math. 24 (1993), 691-703.

[21] J. Schauder, Der Fixpunktsatz in Funktionalr“aumen, Studia Math. 2 (1930), 171-180.

[22] M. Vath, Volterra and integral equations of vector functions, Mercel Dekker (2000). 